\title{
Factors associated with delirium among survivors of acute respiratory distress syndrome: a nationwide cohort study
}

Tak Kyu Oh ${ }^{1,2}$, Hye Youn Park ${ }^{3}$ and In-Ae Song ${ }^{1 *}$

\begin{abstract}
Background: The prevalence of delirium, its associated factors, and its impact on long-term mortality among survivors of acute respiratory distress syndrome (ARDS) is unclear.

Methods: Since this was a population-based study, data were extracted from the National Health Insurance database in South Korea. All adults who were admitted to intensive care units with a diagnosis of ARDS between January 1 , 2010, and December 31, 2019, and who survived for $\geq 60$ days were included. The International Statistical Classification of Diseases and Related Health Problems, tenth revision code of delirium (F05) was used to extract delirium cases during hospitalization.

Results: A total of 6809 ARDS survivors were included in the analysis, and 319 patients (4.7\%) were diagnosed with delirium during hospitalization. In the multivariable logistic regression analysis after covariate adjustment, male sex (odds ratio $[\mathrm{OR}] 1.60,95 \%$ confidence interval $[\mathrm{Cl}] 1.23,2.08 ; P<0.001)$, longer duration of hospitalization (OR 1.02, $95 \% \mathrm{Cl} 1.01,1.03 ; P<0.001)$, neuromuscular blockade use (OR 1.50, 95\% Cl 1.12, 2.01; $P=0.006)$, benzodiazepine (OR $1.55,95 \% \mathrm{Cl} 1.13,2.13 ; P=0.007)$ and propofol $(\mathrm{OR} 1.48,95 \% \mathrm{Cl} 1.01,2.17 ; P=0.046)$ continuous infusion, and concurrent depression ( $\mathrm{OR} 1.31,95 \% \mathrm{Cl} 1.01,1.71 ; P=0.044)$ were associated with a higher prevalence of delirium among ARDS survivors. In the multivariable Cox regression analysis after adjustment for covariates, the occurrence of delirium was not significantly associated with 1-year all-cause mortality, when compared to the other survivors who did not develop delirium (hazard ratio: $0.85,95 \% \mathrm{Cl} 1.01,1.71 ; P=0.044$ ).

Conclusions: In South Korea, 4.7\% of ARDS survivors were diagnosed with delirium during hospitalization in South Korea. Some factors were potential risk factors for the development of delirium, but the occurrence of delirium might not affect 1-year all-cause mortality among ARDS survivors.
\end{abstract}

Keywords: Critical Care, Delirium, Mortality, Respiratory distress syndrome

\section{Background}

Delirium is a serious disturbance in mental abilities that results in an acute confusional status and deteriorating awareness of the environment [1]. It is commonly

\footnotetext{
*Correspondence: songoficu@outlook.kr

1 Department of Anesthesiology and Pain Medicine, Seoul National University Bundang Hospital, Gumi-ro, 173, Beon-gil, Bundang-gu, Seongnam 13620, South Korea

Full list of author information is available at the end of the article
}

diagnosed using the diagnostic and statistical manual of mental disorders, fifth edition (DSM-5) criteria [2]. It occurs frequently among critically ill patients admitted in the intensive care unit (ICU) [3]. Since delirium is a significant risk factor of prolongation of ICU stay and poorer survival outcomes up to one year after discharge among ICU patients $[4,5]$, effective prevention and management of delirium are important. 
For critically ill patients in the ICU, the reported prevalence of delirium is $25-31.8 \%[5,6]$. In particular, critically ill patients who undergo mechanical ventilatory support have a higher risk of delirium; this is an independent predictor of higher 6 -month mortality $[7,8]$. Acute respiratory distress syndrome (ARDS) is characterized by refractory hypoxemia from respiratory failure [9]. Thus, most patients with ARDS require mechanical ventilation, and $36 \%$ of patients requiring mechanical ventilation experienced agitation under light sedation [10]. In addition, ARDS is associated with a higher risk of delirium during ICU stay [11]. However, studies on the relationship between delirium, risk factors, and mortality of patients with ARDS are few. A recent retrospective cohort study reported that $43 \%(124 / 286)$ of patients with ARDS were diagnosed with ICU delirium [12]. In that study, the impact of delirium on mortality of patients with ARDS was not assessed, and the sample size was relatively small [12]. Moreover, the prevalence and impact of delirium on long-term mortality during hospitalization among ARDS survivors have not yet been identified.

Therefore, using a nationwide claim database in South Korea, this study aimed to investigate the prevalence and factors associated with the occurrence of delirium among ARDS survivors. In addition, we examined whether the occurrence of delirium during hospitalization was associated with long-term mortality.

\section{Methods}

\section{Study design and ethical statement}

This study is a nationwide population-based cohort study. It was conducted in accordance with the Strengthening of the Reporting of Observational Studies in Epidemiology guidelines. The study protocol was approved by the Institutional Review Board (IRB) (X-2008-630-903), and the National Health Insurance Service (NHIS) permitted data sharing after approval of the study protocol (NHIS2021-1-424). The requirement of informed consent was waived by the IRB because anonymized data was used in this study.

\section{Data source and study population}

Data (demographic, socioeconomic, and treatment data of all Korean individuals in the sole and public health insurance system in South Korea) was obtained from the NHIS database. All diagnoses of diseases had to be registered by physicians using the International Statistical Classification of Diseases and Related Health Problems, Tenth revision (ICD-10) codes in the NHIS database. The prescription information of all procedures and/or drugs also had to be registered in the NHIS database. These registrations enable patients to receive financial support for treatment expenses by the government.
All adult patients (aged $\geq 18$ years) who were admitted to the ICU for ARDS (J80) between January 1, 2010, and December 31, 2019, were eligible. Patients with a main diagnosis or secondary diagnosis of ARDS were included because ARDS is a clinical syndrome that may occur concurrently with other main diseases such as pneumonia, sepsis, or pancreatitis [13]. The main diagnosis of each patient was determined by the NHIS after hospital discharge or death, as a disease requiring close follow-up or emergency treatment during the patient's hospitalization. If a patient was admitted to the ICU with a diagnosis of $A R D S \geq 2$ times during the study period, only the first ICU admission was considered in this study. To focus on delirium during hospitalization, patients with ARDS who died within 60 days after hospitalization were excluded because 60 -day mortality is a common primary endpoint for patients with severe ARDS [14]. Therefore, patients with ARDS who survived $\geq 60$ days after the diagnosis of ARDS were considered ARDS survivors and included in the final analysis.

\section{Endpoints and outcomes}

The primary endpoint was the diagnosis of delirium during hospitalization and was evaluated from the date of ARDS diagnosis to 60 days after ARDS diagnosis. The ICD-10 code, F05, was used to extract delirium diagnoses. In South Korea, DSM-5 criteria isusually used for diagnosis of delirium in ICU [2], and the registration of ICD-10 codes of delirium enable the financial support of treatment. Moreover, most medical staff in Korean ICUs used Korean version of confusion assessment method for the ICU (CAM-ICU) routinely for screening and assessment of delirium during ICU stays [15]. The ARDS survivors who were diagnosed with delirium constituted the delirium group, while the remaining patients constituted the control group. The secondary endpoint was 1-year all-cause mortality, which was evaluated from the date of ARDS diagnosis to one year after ARDS diagnosis. To better follow up the patients with ARDS for at least one year after ARDS diagnosis, the exact date of death was extracted until December 31, 2020.

First, the prevalence of delirium among ARDS survivors and the factors associated with delirium were investigated. Second, the association between development of delirium during hospitalization among ARDS survivors and 1-year all-cause mortality was examined.

\section{Covariates}

The following variables were considered covariates in this study: age and sex were demographic variables. Household income level and employment status at the time of ARDS treatment was included to reflect patients' socioeconomic status. The patients were 
divided into four groups according to household income levels using quartile ratios. Household income level was registered annually in the NHIS database to determine insurance premiums in South Korea. The admitting department was included as a covariate and classified into two groups (internal medicine and noninternal medicine). Length of hospitalization (day) and total cost of hospitalization (United States dollars) were covariates. Since higher case volume centers are related to better survival outcomes of patients with ARDS [16], the annual case volume of ICU admission for ARDS treatment was considered a covariate. All patients with ARDS were divided into four groups using quartile ratios, based on the hospital in which they were admitted for ARDS treatment: Q1 $\leq 4$; Q2, 5-14; Q3, 15-28; and Q4 $\geq 28$. A main diagnosis of ARDS was considered as a covariate, and diagnoses of shock (R57) or sepsis (A40, A41, and R65.2) were also considered as covariates. If a patient with ARDS had a main diagnosis of sepsis and a secondary diagnosis of ARDS, the diagnosis was sepsis-associated ARDS. To reflect the comorbidity status of patients with ARDS, the Charlson comorbidity index (CCI) was calculated using registered ICD-10 codes within one year before the date of ARDS diagnosis (Additional file 1: Table S1). All patients were divided into four groups according to their CCI scores $(0-1,2-3,4-5$, and $\geq 6)$. Concurrent psychiatric illnesses such as depression (F32, F33, and F34.1), anxiety disorder (F40 and F 41), post-traumatic stress disorder (F43.1), alcohol abuse (F10), and other substance abuse (F11-19) were considered because of the close relationship between concurrent psychiatric disorders and development of delirium among hospitalized patients $[17,18]$. Moreover, underlying disability at admission for ARDS was extracted and considered as a covariate. In South Korea, all disabilities should be registered in the NHIS database to receive various benefits under social welfare programs. The disabilities were divided into two groups such as brain disability and non-brain disability, because delirium is common in patients with neurological disease [19]. Regarding treatment, extracorporeal membrane oxygenation support, neuromuscular blockade use, continuous renal replacement therapy use, duration of mechanical ventilation (day), and cardiopulmonary resuscitation experience were considered as covariates. Information regarding benzodiazepine continuous infusion (midazolam, diazepam, and lorazepam), opioid continuous infusion, and propofol infusion were collected as covariates. Admission to isolated ICU was also collected and considered as a covariate, because isolation in ICU was known as environmental risk factor of delirium in ICU [20].

\section{Statistical analysis}

The clinicopathological characteristics of ARDS survivors are presented as means with standard deviations (SDs) for continuous variables and numbers with percentages for categorical variables. The t-test was used to compare continuous variables, and the chi-square test was used to compare categorical variables between the delirium and control groups. We fitted a multivariable logistic regression model for diagnosing delirium among ARDS survivors. All covariates were included in the model for multivariable adjustment. The Hosmer-Lemeshow test was performed to confirm the goodness of fit of the multivariable model. The results of the logistic regression analysis are presented as odds ratios (ORs) with 95\% confidence intervals (CIs). A multivariable Cox regression model for 1-year all-cause mortality among ARDS survivors was constructed. All covariates were included in the adjusted model. The log-log plot was used to confirm that the central assumption of the Cox proportional hazard model was satisfied. The results of the Cox regression model are presented as hazard ratios (HRs) with 95\% CIs. There was no multicollinearity in both multivariable logistic and Cox regression models as criteria of variance inflation factors: $<2.0$ between all variables. All statistical analyses were performed using $\mathrm{R}$ software (version 4.0.3, $\mathrm{R}$ packages, R Project for Statistical Computing, Vienna, Austria). $P$ values $<0.05$ were considered statistically significant for all analyses.

\section{Results}

A total of 27,889 patients were admitted in the ICU and diagnosed with ARDS in South Korea. After excluding 8,327 patients with $\geq 2$ ICU admissions and 2,459 pediatric patients (below 18 years old), 17,103 adult patients with ARDS were screened initially. Among them, 10, 294 (60.2\%) patients who died within 60 days after ARDS diagnosis were excluded, and 6,809 ARDS survivors were finally included in the analysis. Among them, 319 (4.7\%) patients were diagnosed with delirium during hospitalization (Fig. 1).

The clinicopathological characteristics of the ARDS survivors are presented in Table 1 . The mean age of the ARDS survivors was 66 (SD: 17.2) years, and 61.4\% (4180/6809) of the patients were men. The results of the comparison of clinicopathological characteristics between the delirium and control groups are presented in Table 2. The mean length of hospital stay of the delirium group was longer than that of the control group (30.2 [SD: 21.3 ] vs. 20.2 [SD: 16.8 ] days, respectively; $P<0.001)$. The mean duration of mechanical ventilation in the delirium group was also longer than that in the control group (6.5 [SD: 9.2] vs. 4.5 [SD: 8.8] days, respectively; $P<0.001)$. 
From January 1, 2010, to December 31, 2019

27,889 cases in ICUs for ARDS treatment

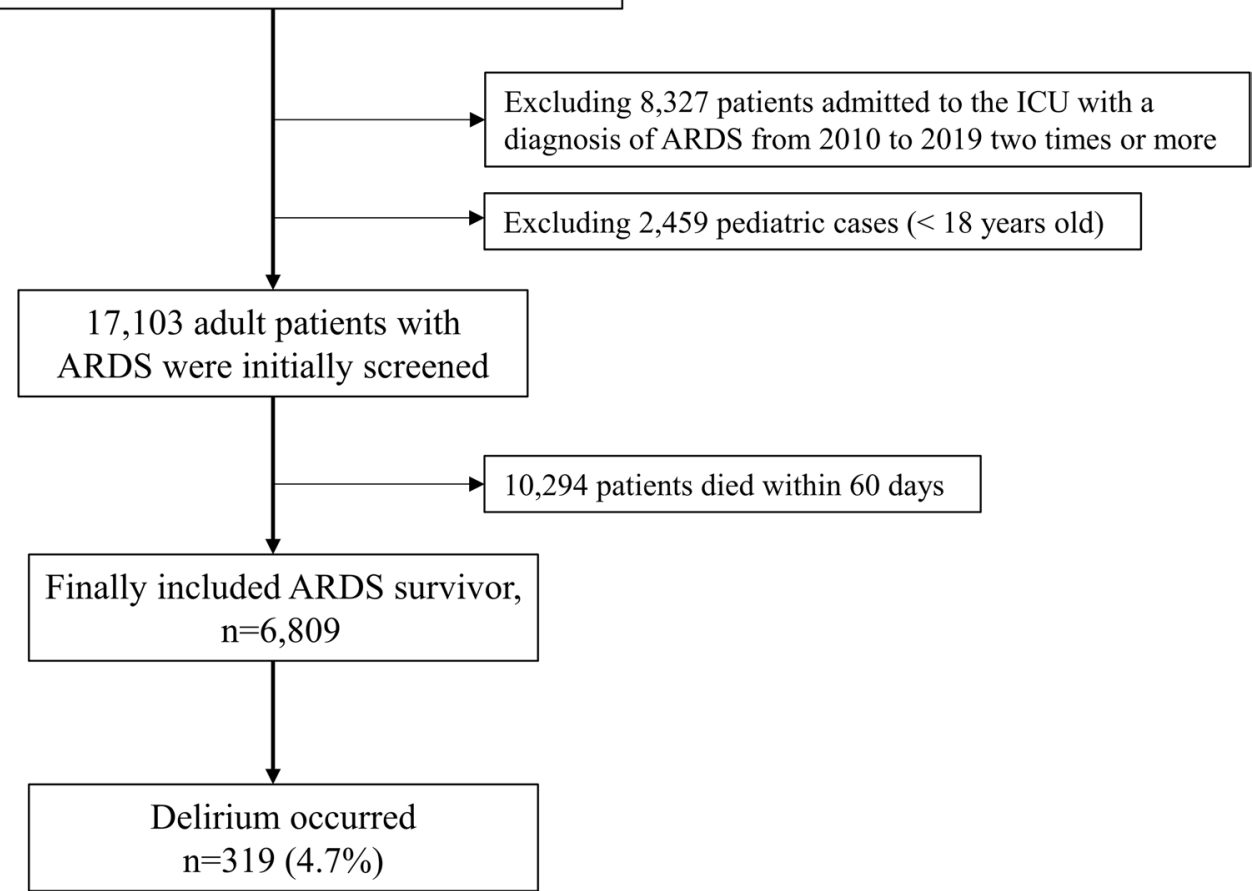

Fig. 1 Selection process flow chart of acute respiratory distress syndrome survivors. ARDS, acute respiratory distress syndrome

The proportion of patients with concurrent depression was higher in the delirium group (34.2\% [109/319]) than in the control group (27.1\% [1757/6490], $P=0.006)$.

Table 3 shows the results of the multivariable logistic regression analysis of the occurrence of delirium among ARDS survivors. Male sex (OR 1.60, 95\% CI 1.23, 2.08; $P<0.001$ ), longer duration of hospitalization (OR 1.02, 95\% CI 1.01, 1.03; $P<0.001$ ), neuromuscular blockade use (OR 1.50, 95\% CI 1.12, 2.01; $P=0.006$ ), benzodiazepine (OR 1.55, 95\% CI 1.13, 2.13; $P=0.007$ ) and propofol (OR 1.48, 95\% CI 1.01, 2.17; $P=0.046)$ continuous infusion, and concurrent depression (OR 1.31, 95\% CI 1.01, 1.71; $P=0.044$ ) were associated with a higher prevalence of delirium among ARDS survivors.

Table 4 shows the results of the multivariable Cox regression analysis for 1-year all-cause mortality among ARDS survivors. Delirium was not associated with 1-year all-cause mortality significantly, when compared to the control group (HR 0.85, 95\% CI 0.69-1.06; $P=0.148$ ).

\section{Discussion}

In this population-based cohort study in South Korea, $4.7 \%$ of ARDS survivors were diagnosed with delirium during hospitalization. Male sex, longer duration of hospitalization, neuromuscular blockade use, benzodiazepine and propofol continuous infusion, and concurrent depression were potential risk factors for the occurrence of delirium in patients with ARDS. However, the occurrence of delirium was not associated with the 1-year all-cause mortality in this study. Our results suggest that although ARDS survivors experienced delirium during hospitalization, delirium did not affect the relative long-term survival after hospital discharge.

A recent cohort study by Kalra et al. reported that $43 \%$ (124/286) of patients with ARDS were diagnosed with ICU delirium [12], which is a much higher prevalence than that in our study. Several reasons might explain this difference. First, we included ARDS survivors who were alive for $\geq 60$ days after ARDS diagnosis, while Kalra et al. included all patients diagnosed with ARDS using the Berlin definition in their study [12]. Patients with ARDS who were diagnosed with delirium and died within 60 days after the diagnosis of delirium were excluded from our study. Second, the CAM-ICU assessment tool was used to define delirium in the study by Kalra et al. [12], whereas we used registered ICD-10 codes in a large population. Therefore, some cases may have been missed in our study due to variations in delirium diagnosis in each hospital in South Korea. 
Table 1 The clinicopathological characteristics of the ARDS survivors

\begin{tabular}{|c|c|}
\hline Variable & Mean (SD) or $\mathrm{N}(\%)$ \\
\hline Age, year & $66.0(17.2)$ \\
\hline Sex, male & $4180(61.4)$ \\
\hline Having a job at admission for ARDS & $3555(52.2)$ \\
\hline \multicolumn{2}{|l|}{ Annual income level at ARDS treatment } \\
\hline Q1 (Lowest) & $2031(29.8)$ \\
\hline Q2 & $1073(15.8)$ \\
\hline Q3 & $1333(19.6)$ \\
\hline Q4 (Highest) & $2227(32.7)$ \\
\hline Unknown & $145(2.1)$ \\
\hline \multicolumn{2}{|l|}{ Admitting department } \\
\hline IM & $5480(80.5)$ \\
\hline Non-IM & $1329(19.5)$ \\
\hline Length of hospitalization, day & $20.7(17.2)$ \\
\hline Total cost for hospitalization, USD & $10,799.2(13,605.4)$ \\
\hline \multicolumn{2}{|l|}{ Annual case volume of ARDS admission } \\
\hline $\mathrm{Q} 1 \leq 4$ & $1417(20.8)$ \\
\hline Q2: 5-14 & $1648(24.2)$ \\
\hline Q3: 15-28 & $1966(28.9)$ \\
\hline $\mathrm{Q} 4 \geq 28$ & $1778(26.1)$ \\
\hline Main diagnosis of ARDS & $3390(49.8)$ \\
\hline Sepsis associated ARDS & $393(5.3)$ \\
\hline Diagnosis of shock during hospitalization & $363(5.3)$ \\
\hline Underlying brain disability & $484(7.1)$ \\
\hline Underlying non-brain disability & $1480(21.7)$ \\
\hline Isolated ICU admission & $196(2.9)$ \\
\hline \multicolumn{2}{|l|}{$\mathrm{CCl}$ at hospital admission for ARDS } \\
\hline $0-1$ & $1523(22.4)$ \\
\hline $2-3$ & $2171(31.9)$ \\
\hline $4-5$ & $1558(22.9)$ \\
\hline$\geq 6$ & $1557(22.9)$ \\
\hline ECMO support & $317(4.7)$ \\
\hline NMB use & $2238(32.9)$ \\
\hline CRRT use & $299(4.4)$ \\
\hline Benzodiazepine continuous infusion & $3889(57.1)$ \\
\hline Opioid continuous infusion & $1049(15.4)$ \\
\hline Propofol continuous infusion & $560(8.2)$ \\
\hline Duration of Mechanical Ventilator use, day & $4.6(8.8)$ \\
\hline Experience of CPR during hospitalization & $236(3.5)$ \\
\hline \multicolumn{2}{|l|}{ Concurrent other psychiatric illness } \\
\hline Depression & $1866(27.4)$ \\
\hline Anxiety disorder & $2324(34.1)$ \\
\hline PTSD & $11(0.2)$ \\
\hline Alcohol abuse & $314(4.6)$ \\
\hline Other substance abuse & $36(0.5)$ \\
\hline \multicolumn{2}{|l|}{ Year of admission for ARDS } \\
\hline 2010 & $849(12.5)$ \\
\hline 2011 & $656(9.6)$ \\
\hline 2012 & $607(8.9)$ \\
\hline 2013 & $527(7.7)$ \\
\hline
\end{tabular}

Table 1 (continued)

\begin{tabular}{cl}
\hline Variable & Mean (SD) or N (\%) \\
\hline 2014 & $641(9.4)$ \\
2015 & $621(9.1)$ \\
2016 & $831(12.2)$ \\
2017 & $705(10.4)$ \\
2018 & $723(10.6)$ \\
2019 & $649(9.5)$ \\
1 -year all-cause mortality & $1868(27.4)$ \\
\hline
\end{tabular}

ARDS, acute respiratory distress syndrome; SD, standard deviation; IM, internal medicine; USD, United States Dollar; CCI, Charlson comorbidity index; ECMO, extracorporeal membrane oxygenation; NMB, neuromuscular blockade; CRRT, continuous renal replacement therapy; CPR, cardiopulmonary resuscitation; PTSD, post-traumatic stress disorder

Male sex has been a known risk factor for delirium in hospitalized elderly patients [21, 22]. Previous studies have reported that male sex is also a risk factor for the development of delirium after hip fracture surgery [23] and vascular surgery [24]. However, in a recent review, sex was not associated with the risk of delirium in adult ICU patients [25]. Although the biological mechanism of the relationship between sex and delirium remains unclear, sex differences in immune responses to stressors may explain this mechanism [26, 27]. Inflammation plays a major role in the pathogenesis of delirium [28]. A previous study reported that men had a higher C-reactive protein response to an endotoxin challenge than women [26]. However, another previous study reported contradictory findings: pro-inflammatory and innate immune responses were higher among women [27]. Therefore, the effect of sex on the development of delirium in critically ill patients such as patients with ARDS needs to be confirmed in future studies.

In our study, concurrent ARDS was a risk factor for the development of delirium among ARDS survivors; this was consistent with the results of the study by Kalra et al. [12]. Concurrent depression is an important risk factor of delirium among elderly and hospitalized patients [29]. Moreover, concurrent depression is known to be interrelated with delirium and to have a clinical overlap with delirium among elderly people [18]. However, in our study, the concurrent anxiety disorder and PTSD were not associated with the development of delirium among ARDS survivors. Meanwhile, Kalra et al. reported that concurrent anxiety disorder was associated with delirium in patients with ARDS [12]. Thus, future studies on the risk factors of delirium among patients with ARDS should consider the various types of mental illness.

The increased association of sedative use (benzodiazepine or propofol) with delirium in patients with ARDS was an important finding. Although patients 
Table 2 The comparison of clinicopathological characteristics between the delirium and control groups

\begin{tabular}{|c|c|c|c|}
\hline Variable & $\begin{array}{l}\text { Delirium group } \\
n=319\end{array}$ & $\begin{array}{l}\text { Control group } \\
n=6,490\end{array}$ & $P$ value \\
\hline Age, year & $66.7(17.3)$ & $66.0(17.2)$ & 0.414 \\
\hline Sex, male & $232(72.7)$ & $3948(60.8)$ & $<0.001$ \\
\hline Having a job at admission for ARDS & $175(54.9)$ & $3380(52.1)$ & 0.332 \\
\hline Annual income level at ARDS treatment & & & 0.510 \\
\hline Q1 (Lowest) & $92(28.8)$ & $1939(29.9)$ & \\
\hline Q2 & $50(15.7)$ & $1023(15.8)$ & \\
\hline Q3 & $70(21.9)$ & $1263(19.5)$ & \\
\hline Q4 (Highest) & $104(32.6)$ & $2123(32.7)$ & \\
\hline Unknown & $3(0.9)$ & $142(2.2)$ & \\
\hline Admitting department: IM & $273(85.6)$ & $5207(80.2)$ & 0.019 \\
\hline Length of hospitalization, day & $30.2(21.3)$ & $20.2(16.8)$ & $<0.001$ \\
\hline Total cost for hospitalization, USD & $18,394.5(17,772.2)$ & $10,425.9(13,257.2)$ & $<0.001$ \\
\hline Underlying brain disability & $14(4.4)$ & $470(7.2)$ & 0.068 \\
\hline Underlying non-brain disability & $74(23.2)$ & $1406(21.7)$ & 0.517 \\
\hline Annual case volume of ARDS admission & & & $<0.0012$ \\
\hline $\mathrm{Q} 1 \leq 4$ & $38(11.9)$ & $1379(21.2)$ & \\
\hline Q2: 5-14 & $64(20.1)$ & $1584(24.4)$ & \\
\hline Q3: 15-28 & $100(31.3)$ & $1866(28.8)$ & \\
\hline $\mathrm{Q} 4 \geq 28$ & $117(36.7)$ & $1661(25.6)$ & \\
\hline Main diagnosis of ARDS & $162(50.8)$ & $3228(49.7)$ & 0.715 \\
\hline Sepsis associated ARDS & $37(11.6)$ & $796(12.3)$ & 0.723 \\
\hline Diagnosis of shock during hospitalization & $23(7.2)$ & $340(5.2)$ & 0.126 \\
\hline Isolated ICU admission & $13(4.1)$ & $183(2.8)$ & 0.190 \\
\hline $\mathrm{CCl}$ at hospital admission for ARDS & & & 0.584 \\
\hline $0-1$ & $62(19.4)$ & $1461(22.5)$ & \\
\hline $2-3$ & $102(32.0)$ & 2069 (31.9) & \\
\hline $4-5$ & $76(23.8)$ & $1482(22.8)$ & \\
\hline$\geq 6$ & $79(24.8)$ & $1478(22.8)$ & \\
\hline ECMO support & $23(7.2)$ & $294(4.5)$ & 0.027 \\
\hline NMBA use & $171(53.6)$ & $2067(31.8)$ & $<0.001$ \\
\hline CRRT use & $20(6.3)$ & $279(4.3)$ & 0.094 \\
\hline Benzodiazepine continuous infusion & $248(77.7)$ & $3641(56.1)$ & $<0.001$ \\
\hline Opioid continuous infusion & $77(24.1)$ & $972(15.0)$ & $<0.001$ \\
\hline Propofol continuous infusion & $51(16.0)$ & $509(7.8)$ & $<0.001$ \\
\hline Duration of Mechanical Ventilator use, day & $6.5(9.2)$ & $4.5(8.8)$ & $<0.001$ \\
\hline Experience of CPR during hospitalization & $16(5.0)$ & $220(3.4)$ & 0.121 \\
\hline Concurrent depression & $109(34.2)$ & $1757(27.1)$ & 0.006 \\
\hline Concurrent anxiety disorder & $119(37.3)$ & $2205(34.0)$ & 0.221 \\
\hline Concurrent PTSD & $1(0.3)$ & $10(0.2)$ & 0.489 \\
\hline \multicolumn{4}{|l|}{ Concurrent alcohol abuse } \\
\hline \multicolumn{4}{|l|}{ Concurrent other substance abuse } \\
\hline Year of admission for ARDS & & & 0.099 \\
\hline 2010 & $28(8.8)$ & $821(12.7)$ & \\
\hline 2011 & $19(6.0)$ & $637(9.8)$ & \\
\hline 2012 & $29(9.1)$ & $578(8.9)$ & \\
\hline 2013 & $34(10.7)$ & $493(7.6)$ & \\
\hline 2014 & $33(10.3)$ & $608(9.4)$ & \\
\hline 2015 & $34(10.7)$ & $587(9.0)$ & \\
\hline 2016 & $43(13.5)$ & 788 (12.1) & \\
\hline
\end{tabular}


Table 2 (continued)

\begin{tabular}{lll}
\hline Variable & $\begin{array}{l}\text { Delirium group } \\
\boldsymbol{n}=\mathbf{3 1 9}\end{array}$ & $\begin{array}{l}\text { Control group } \\
\boldsymbol{n}=\mathbf{6 , 4 9 0}\end{array}$ \\
\hline 2017 & $32(10.0)$ & $673(10.4)$ \\
2018 & $39(12.2)$ & $684(10.54)$ \\
2019 & $28(8.8)$ & $621(9.6)$ \\
1 -year all-cause mortality & $92(28.8)$ & $1776(27.4)$ \\
\hline
\end{tabular}

Presented as means with standard deviations for continuous variables and numbers with percentages for categorical variables

ARDS, acute respiratory distress syndrome; IM, internal medicine; USD, United States Dollar; CCI, Charlson comorbidity index; ECMO, extracorporeal membrane oxygenation; NMB, neuromuscular blockade; CRRT, continuous renal replacement therapy; CPR, cardiopulmonary resuscitation; PTSD, post-traumatic stress disorder

Table 3 Multivariable logistic regression analysis of the occurrence of delirium among ARDS survivors

\begin{tabular}{|c|c|c|}
\hline Variable & OR $(95 \% \mathrm{Cl})$ & $P$ value \\
\hline Age, year & $1.01(1.00,1.02)$ & 0.072 \\
\hline Sex, male (vs female) & $1.60(1.23,2.08)$ & $<0.001$ \\
\hline Having a job at admission for ARDS & $1.08(0.85,1.38)$ & 0.511 \\
\hline \multicolumn{3}{|l|}{ Annual income level at ARDS treatment } \\
\hline Q2 (vs Q1: Lowest) & $0.95(0.66,1.38)$ & 0.795 \\
\hline Q3 (vs Q1: Lowest) & $1.09(0.78,1.52)$ & 0.629 \\
\hline Q4: Highest (vs Q1: Lowest) & $0.86(0.63,1.17)$ & 0.344 \\
\hline Unknown (vs Q1: Lowest) & $0.46(0.14,1.49)$ & 0.195 \\
\hline Admitting department: IM (vs non-IM) & $1.44(1.02,2.02)$ & 0.037 \\
\hline Length of hospitalization, day & $1.02(1.01,1.03)$ & $<0.001$ \\
\hline Total cost for hospitalization, 1000 USD & $1.01(0.99,1.02)$ & 0.333 \\
\hline Underlying brain disability & $0.65(0.37,1.14)$ & 0.129 \\
\hline Underlying non-brain disability & $0.99(0.74,1.31)$ & 0.924 \\
\hline \multicolumn{3}{|l|}{ Annual case volume of ARDS admission } \\
\hline Q2: 5-14 (vs Q1 $\leq 4)$ & $1.06(0.69,1.61)$ & 0.805 \\
\hline Q3: 15-28 (vs Q1 $\leq 4)$ & $1.32(0.88,1.98)$ & 0.176 \\
\hline $\mathrm{Q} 4 \geq 28($ vs Q1 $\leq 4)$ & $1.79(1.20,2.69)$ & 0.005 \\
\hline $\begin{array}{l}\text { Main diagnosis of ARDS (vs Secondary diag- } \\
\text { nosis of ARDS) }\end{array}$ & $0.94(0.74,1.19)$ & 0.609 \\
\hline Sepsis associated ARDS & $0.67(0.46,0.96)$ & 0.030 \\
\hline Diagnosis of shock during hospitalization & $1.02(0.65,1.61)$ & 0.927 \\
\hline Isolated ICU admission & $0.99(0.53,1.85)$ & 0.981 \\
\hline \multicolumn{3}{|l|}{$\mathrm{CCl}$ at hospital admission for ARDS } \\
\hline $2-3$ (vs $0-1)$ & $1.01(0.72,1.41)$ & 0.961 \\
\hline $4-5$ (vs $0-1)$ & $0.96(0.67,1.38)$ & 0.830 \\
\hline$\geq 6$ (vs $0-1$ ) & $0.95(0.66,1.36)$ & 0.772 \\
\hline ECMO support & $0.98(0.57,1.67)$ & 0.930 \\
\hline NMB use & $1.50(1.12,2.01)$ & 0.006 \\
\hline CRRT use & $0.84(0.50,1.40)$ & 0.503 \\
\hline Benzodiazepine continuous infusion & $1.55(1.13,2.13)$ & 0.007 \\
\hline Opioid continuous infusion & $1.05(0.73,1.53)$ & 0.781 \\
\hline Propofol continuous infusion & $1.48(1.01,2.17)$ & 0.046 \\
\hline Duration of Mechanical Ventilator use, day & $0.99(0.98,1.00)$ & 0.050 \\
\hline Experience of CPR during hospitalization & $1.01(0.58 ., 1.75()$ & 0.973 \\
\hline Concurrent anxiety disorder & $1.12(0.87,1.44)$ & 0.385 \\
\hline Concurrent depression & $1.33(1.03,1.73)$ & 0.031 \\
\hline Concurrent PTSD & $2.25(0.27,18.42)$ & 0.451 \\
\hline
\end{tabular}

Table 3 (continued)

\begin{tabular}{lll}
\hline Variable & OR $(95 \%$ Cl) & $P$ value \\
\hline Concurrent alcohol abuse & & \\
Concurrent other substance abuse & & \\
Year of admission for ARDS & $1.41(0.82,1.54)$ & 0.572 \\
2011 (vs 2010) & $1.41(0.82,2.43)$ & 0.210 \\
2012 (vs 2010) & $1.57(0.93,2.67)$ & 0.094 \\
2013 (vs 2010) & $1.24(0.73,2.11)$ & 0.421 \\
2014 (vs 2010) & $1.41(0.84,2.39)$ & 0.198 \\
2015 (vs 2010) & $1.41(0.85 ., 2.34)$ & 0.179 \\
2016 (vs 2010) & $1.17(0.69,2.00)$ & 0.558 \\
2017 (vs 2010) & $1.38(0.82,2.32)$ & 0.226 \\
2018 (vs 2010) & $1.03(0.59,1.81)$ & 0.921 \\
2019 (vs 2010) & &
\end{tabular}

Hosmer Lemeshow, chi-square: $4.88, d f=8, P=0.770$

ARDS, acute respiratory distress syndrome; $\mathrm{OR}$, odds ratio; $\mathrm{Cl}$, confidence interval; IM, internal medicine; USD, United States Dollar; $\mathrm{CCl}$, Charlson comorbidity index; ECMO, extracorporeal membrane oxygenation; $\mathrm{NMB}_{\text {, }}$ neuromuscular blockade; CRRT, continuous renal replacement therapy; CPR, cardiopulmonary resuscitation; PTSD, post-traumatic stress disorder

with ARDS usually require deep sedation for mechanical ventilation, the protocol-based light sedation strategies had been suggested based on previous reports [30]. Hager et al. reported that reducing deep sedation was associated with decreased delirium in patients with acute lung injury [31]. Therefore, the deep sedation using propofol or benzodiazpine might increase risk of delirium in patients with ARDS in studies. Moreover, it was also possible that patients who received deep sedation using propofol or benzodiazepine might be more severe than patients with ARDS, and the risk of delirium was higher than other patients with ARDS. As the relationship between depth of sedation and risk of delirium among mechanically ventilated patients remains controversial and inconclusive issue [32], more research is needed regarding this issue.

Interestingly, there was no significant association between the occurrence of delirium during hospitalization and 1-year mortality among ARDS survivors in this study. There are previous studies on the association 
Table 4 Multivariable Cox regression analysis for 1-year all-cause mortality among ARDS survivors

\begin{tabular}{|c|c|c|}
\hline Variable & $\mathrm{HR}(95 \% \mathrm{Cl})$ & $P$ value \\
\hline Delirium group (vs control) & $0.85(0.69,1.06)$ & 0.148 \\
\hline Age, year & $1.04(1.04,1.05)$ & $<0.001$ \\
\hline Sex, male (vs female) & $1.43(1.29,1.58)$ & $<0.001$ \\
\hline Having a job at admission for ARDS & $1.01(0.91,1.11)$ & 0.908 \\
\hline \multicolumn{3}{|l|}{ Annual income level at ARDS treatment } \\
\hline Q2 (vs Q1: Lowest) & $0.86(0.74,1.01)$ & 0.065 \\
\hline Q3 (vs Q1: Lowest) & $0.93(0.80,1.07)$ & 0.278 \\
\hline Q4: Highest (vs Q1: Lowest) & $0.95(0.85,1.07)$ & 0.411 \\
\hline Unknown (vs Q1: Lowest) & $0.96(0.71,1.31)$ & 0.814 \\
\hline Admitting department: IM (vs non-IM) & $0.84(0.75,0.95)$ & 0.005 \\
\hline Length of hospitalization, day & $1.01(1.00,1.01)$ & $<0.001$ \\
\hline Total cost for hospitalization, 1000 USD & $1.02(1.01,1.02)$ & $<0.001$ \\
\hline Underlying brain disability & $1.25(1.07,1.46)$ & 0.006 \\
\hline Underlying non-brain disability & $1.13(1.01,1.26)$ & 0.028 \\
\hline \multicolumn{3}{|l|}{ Annual case volume of ARDS admission } \\
\hline Q2: 5-14 (vs Q1 $\leq 4)$ & $0.85(0.74,0.98)$ & 0.021 \\
\hline Q3: 15-28 (vs Q1 <4) & $0.98(0.86,1.12)$ & 0.809 \\
\hline $\mathrm{Q} 4 \geq 28$ (vs Q1 $\leq 4)$ & $0.93(0.80,1.07)$ & 0.289 \\
\hline $\begin{array}{l}\text { Main diagnosis of ARDS (vs Secondary diagno- } \\
\text { sis of ARDS) }\end{array}$ & $0.91(0.83,1.00)$ & 0.041 \\
\hline Sepsis associated ARDS & $1.10(0.96,1.26)$ & 0.168 \\
\hline Diagnosis of shock during hospitalization & $0.99(0.80,1.23)$ & 0.945 \\
\hline Isolated ICU admission & $0.87(0.64,1.17)$ & 0.346 \\
\hline \multicolumn{3}{|l|}{$\mathrm{CCl}$ at hospital admission for ARDS } \\
\hline $2-3$ (vs $0-1)$ & $1.17(1.01,1.36)$ & 0.043 \\
\hline $4-5$ (vs $0-1)$ & $1.54(1.32,1.80)$ & $<0.001$ \\
\hline$\geq 6$ (vs $0-1)$ & $2.08(1.79,2.41)$ & $<0.001$ \\
\hline ECMO support & $0.66(0.47,0.92)$ & 0.014 \\
\hline NMBA use & $0.70(0.62,0.80)$ & $<0.001$ \\
\hline CRRT use & $0.90(0.71,1.16)$ & 0.425 \\
\hline Benzodiazepine continuous infusion & $1.03(0.92,1.15)$ & 0.600 \\
\hline Opioid continuous infusion & $0.75(0.63,0.89)$ & 0.001 \\
\hline Propofol continuous infusion & $0.93(0.75,1.16)$ & 0.526 \\
\hline Duration of Mechanical Ventilator use, day & $1.01(1.01,1.02)$ & $<0.001$ \\
\hline Experience of CPR during hospitalization & $1.80(1.46,2.22)$ & $<0.001$ \\
\hline Concurrent anxiety disorder & $0.89(0.80,0.98)$ & 0.020 \\
\hline Concurrent depression & $1.16(1.05,1.29)$ & 0.005 \\
\hline Concurrent PTSD & $0.41(0.06,2.96)$ & 0.379 \\
\hline Concurrent alcohol abuse & $0.89(0.69,1.15)$ & 0.383 \\
\hline Concurrent other substance abuse & $0.63(0.28,1.41)$ & 0.260 \\
\hline \multicolumn{3}{|l|}{ Year of admission for ARDS } \\
\hline 2011 (vs 2010) & $1.06(0.88,1.29)$ & 0.524 \\
\hline 2012 (vs 2010) & $0.99(0.82,1.21)$ & 0.928 \\
\hline 2013 (vs 2010) & $1.13(0.93,1.38)$ & 0.227 \\
\hline 2014 (vs 2010) & $0.94(0.77,1.14)$ & 0.522 \\
\hline 2015 (vs 2010) & $0.97(0.80,1.17)$ & 0.739 \\
\hline 2016 (vs 2010) & $0.97(0.80,1.17)$ & 0.720 \\
\hline 2017 (vs 2010) & $0.88(0.72,1.08)$ & 0.214 \\
\hline 2018 (vs 2010) & $0.91(0.74,1.12)$ & 0.395 \\
\hline 2019 (vs 2010) & $0.80(0.64,0.99)$ & 0.039 \\
\hline
\end{tabular}

Table 4 (continued)

ARDS, acute respiratory distress syndrome; $\mathrm{HR}$, hazard ratio; $\mathrm{Cl}$, confidence interval; IM, internal medicine; USD, United States Dollar; CCl, Charlson comorbidity index; ECMO, extracorporeal membrane oxygenation; $\mathrm{NMB}$, neuromuscular blockade; CRRT, continuous renal replacement therapy; CPR, cardiopulmonary resuscitation; PTSD, post-traumatic stress disorder

between the occurrence of delirium and 1-year mortality [4, 33-35]. Pisani et al. reported that the duration of delirium was associated with increased 1-year all-cause mortality in older ICU patients [4]. Contrarily, Wolters et al. reported that delirium during ICU stay was not associated with 1-year mortality among survivors of a critical illness in the Netherlands [36], which is similar to the results of our study. The varying characteristics of the study populations (overall ICU population and survivors) of the two studies might explain the differences $[4,36]$. Wolters et al. excluded ICU patients who died during their ICU stay [36], and this was consistent with our study. In contrast, Pisani et al. did not exclude ICU patients who died during their ICU stay or hospitalization [4]. The occurrence of delirium during hospitalization has been reported to increase hospital mortality among critically ill patients [37]; therefore, we excluded patients with ARDS who had more severe conditions, who might have been diagnosed with delirium, and died during hospitalization. The exclusion of the patients who died within 60 days of ARDS diagnosis may have influenced the results.

This study had several limitations. First, the severity of ARDS was not assessed accurately. For example, the $\mathrm{PaO} 2 / \mathrm{FiO} 2$ ratio (ratio of the patient's oxygen in arterial blood $[\mathrm{PaO} 2]$ to the fraction of oxygen in inspired air [FiO2]) and Acute Physiology and Chronic Health Evaluation II scores were not considered in this study for accurate adjustment of ARDS severity. Second, some treatment information, such as prone positioning, was not considered in this study because there is no prescription code for prone positioning in South Korea. Third, the model was not adjusted for important variables such as smoking history, pulmonary function test results, and body mass index. This is because their data were unavailable in the NHIS database. Lastly, the CAM-ICU, which is known to be the best measuring tool for the evaluation of delirium, was not used in this study due to unavailable data in the NHIS database. Therefore, some cases may have been missed or inaccurate diagnoses of delirium may have been made: these may also have affected the results. Moreover, we cannot guarantee that all patients with delirium were diagnosed and treated using the DSM-5 criteria in this study [2]. In these perspectives, the methodology using ICD-10 code of delirium made our study include obvious and serious cases that required immediate treatment of delirium. This limitation 
regarding methodology using ICD-10 code should be considered the prevalence of delirium and associated factor in this study.

\section{Conclusions}

In conclusion, $4.7 \%$ of ARDS survivors were diagnosed with delirium during hospitalization in South Korea. Male sex, longer duration of hospitalization, neuromuscular blockade use, benzodiazepine and propofol continuous infusion, and concurrent depression were potential risk factors for the development of delirium, but the occurrence of delirium might not affect 1-year all-cause mortality among ARDS survivors.

\section{Abbreviations}

ARDS: Acute respiratory distress syndrome; CAM-ICU: Confusion assessment method for the ICU; CCl: Charlson comorbidity index; Cl: Confidence intervals; DSM-5: Diagnostic and statistical manual of mental disorders, fifth edition; HR: Hazard ratios; ICU: Intensive care unit; IRB: Institutional Review Board; OR: Odds ratios; SD: Standard deviation.

\section{Supplementary Information}

The online version contains supplementary material available at https://doi. org/10.1186/s12890-021-01714-0.

Additional file 1 Table S1. The ICD-10 codes used by comorbidity to compute the Charlson comorbidity index

\section{Acknowledgements}

None

\section{Authors' contributions}

TKO and I-AS: designed the study, analyzed the data, interpreted the data, drafted the manuscript, HYP: interpreted the data. Methodology, and reviewed the manuscript. All authors approved the final version of manuscript. All authors read and approved the final manuscript.

\section{Funding}

This research did not receive any specific grant from funding agencies in the public, commercial, or not-for-profit sectors.

\section{Availability of data and materials}

All data will be available upon reasonable request to corresponding author.

\section{Declarations}

\section{Ethics approval and consent to participate}

The study protocol was approved by the Institutional Review Board (IRB) (X-2008-630-903), and the National Health Insurance Service (NHIS) permitted data sharing after approval of the study protocol (NHIS-2021-1-424). The requirement of informed consent was waived by the IRB because anonymized data was used in this study.

\section{Consent for publication}

Not applicable.

\section{Competing interests}

The authors declare that they have no competing interests.

\section{Author details}

'Department of Anesthesiology and Pain Medicine, Seoul National University Bundang Hospital, Gumi-ro, 173, Beon-gil, Bundang-gu, Seongnam 13620,
South Korea. ${ }^{2}$ Department of Anesthesiology and Pain Medicine, College of Medicine, Seoul National University, Seoul, South Korea. ${ }^{3}$ Department of Psychiatry, Seoul National University Bundang Hospital, Seongnam, South Korea.

Received: 4 September 2021 Accepted: 29 October 2021

Published online: 01 November 2021

\section{References}

1. Marcantonio ER. Delirium in hospitalized older adults. N Engl J Med. 2017;377(15):1456-66.

2. European Delirium A, American Delirium S. The DSM-5 criteria, level of arousal and delirium diagnosis: inclusiveness is safer. BMC Med. 2014;12:141.

3. Choi JG. Delirium in the intensive care unit. Korean J Anesthesiol. 2013;65(3):195-202.

4. Pisani MA, Kong SY, KasI SV, Murphy TE, Araujo KL, Van Ness PH. Days of delirium are associated with 1-year mortality in an older intensive care unit population. Am J Respir Crit Care Med. 2009;180(11):1092-7.

5. Salluh Jl, Wang H, Schneider EB, Nagaraja N, Yenokyan G, Damluji A, Serafim RB, Stevens RD. Outcome of delirium in critically ill patients: systematic review and meta-analysis. BMJ. 2015;350:h2538.

6. Collet MO, Caballero J, Sonneville R, Bozza FA, Nydahl P, Schandl A, Woien $\mathrm{H}$, Citerio G, van den Boogaard M, Hastbacka J, et al. Prevalence and risk factors related to haloperidol use for delirium in adult intensive care patients: the multinational AID-ICU inception cohort study. Intensive Care Med. 2018;44(7):1081-9.

7. Ely EW, Inouye SK, Bernard GR, Gordon S, Francis J, May L, Truman B, Speroff T, Gautam S, Margolin R, et al. Delirium in mechanically ventilated patients: validity and reliability of the confusion assessment method for the intensive care unit (CAM-ICU). JAMA. 2001;286(21):2703-10.

8. Ely EW, Shintani A, Truman B, Speroff T, Gordon SM, Harrell FE Jr, Inouye SK, Bernard GR, Dittus RS. Delirium as a predictor of mortality in mechanically ventilated patients in the intensive care unit. JAMA. 2004;291(14):1753-62.

9. Rawal G, Yadav S, Kumar R. Acute respiratory distress syndrome: an update and review. J Transl Int Med. 2018;6(2):74-7.

10. Zhang Z, Liu J, Xi J, Gong Y, Zeng L, Ma P. Derivation and validation of an ensemble model for the prediction of agitation in mechanically ventilated patients maintained under light sedation. Crit Care Med. 2021;49(3):e279-90.

11. Hsieh SJ, Soto GJ, Hope AA, Ponea A, Gong MN. The association between acute respiratory distress syndrome, delirium, and in-hospital mortality in intensive care unit patients. Am J Respir Crit Care Med. 2015;191(1):71-8.

12. Kalra SS, Jaber J, Alzghoul BN, Hyde R, Parikh S, Urbine D, Reddy R. Preexisting psychiatric illness is associated with an increased risk of delirium in patients with acute respiratory distress syndrome. J Intensive Care Med 2021;8850666211019009.

13. Fujishima S. Pathophysiology and biomarkers of acute respiratory distress syndrome. J Intensive Care. 2014;2(1):32.

14. Combes A, Hajage D, Capellier G, Demoule A, Lavoue S, Guervilly C, Da Silva D, Zafrani L, Tirot P, Veber B, et al. Extracorporeal membrane oxygenation for severe acute respiratory distress syndrome. N Engl J Med. 2018;378(21):1965-75.

15. Heo EY, Lee BJ, Hahm BJ, Song EH, Lee HA, Yoo CG, Kim YW, Han SK, Shim YS, Lee SM. Translation and validation of the Korean confusion assessment method for the intensive care unit. BMC Psychiatry. 2011;11:94.

16. Ike JD, Kempker JA, Kramer MR, Martin GS. The association between acute respiratory distress syndrome hospital case volume and mortality in a U.S. Cohort, 2002-2011. Crit Care Med. 2018;46(5):764-73.

17. Ojeahere MI, de Filippis R, Ransing R, Karaliuniene R, Ullah I, Bytyci DG, Abbass Z, Kilic O, Nahidi M, Hayatudeen N, et al. Management of psychiatric conditions and delirium during the COVID-19 pandemic across continents: lessons learned and recommendations. Brain Behav Immun Health. 2020;9:100147.

18. O'Sullivan R, Inouye SK, Meagher D. Delirium and depression: interrelationship and clinical overlap in elderly people. Lancet Psychiatry. 2014;1(4):303-11. 
19. Hufner K, Sperner-Unterweger B. Delirium in patients with neurological diseases: diagnosis, management and prognosis. Nervenarzt. 2014;85(4):427-36.

20. Van Rompaey B, Elseviers MM, Schuurmans MJ, Shortridge-Baggett LM, Truijen S, Bossaert L. Risk factors for delirium in intensive care patients: a prospective cohort study. Crit Care. 2009;13(3):R77.

21. Schor JD, Levkoff SE, Lipsitz LA, Reilly CH, Cleary PD, Rowe JW, Evans DA. Risk factors for delirium in hospitalized elderly. JAMA. 1992;267(6):827-31.

22. Kim H, Chung S, Joo YH, Lee JS. The major risk factors for delirium in a clinical setting. Neuropsychiatr Dis Treat. 2016;12:1787-93.

23. Oh ES, Sieber FE, Leoutsakos JM, Inouye SK, Lee HB. Sex differences in hip fracture surgery: preoperative risk factors for delirium and postoperative outcomes. J Am Geriatr Soc. 2016;64(8):1616-21.

24. Galyfos GC, Geropapas GE, Sianou A, Sigala F, Filis K. Risk factors for postoperative delirium in patients undergoing vascular surgery. J Vasc Surg. 2017;66(3):937-46.

25. Krewulak KD, Stelfox HT, Ely EW, Fiest KM. Risk factors and outcomes among delirium subtypes in adult ICUs: a systematic review. J Crit Care. 2020;56:257-64.

26. Ferguson JF, Patel PN, Shah RY, Mulvey CK, Gadi R, Nijjar PS, Usman HM, Mehta NN, Shah R, Master SR, et al. Race and gender variation in response to evoked inflammation. J Transl Med. 2013;11:63.

27. van Eijk LT, Dorresteijn MJ, Smits P, van der Hoeven JG, Netea MG, Pickkers $P$. Gender differences in the innate immune response and vascular reactivity following the administration of endotoxin to human volunteers. Crit Care Med. 2007;35(6):1464-9.

28. Maclullich AM, Ferguson KJ, Miller T, de Rooij SE, Cunningham C. Unravelling the pathophysiology of delirium: a focus on the role of aberrant stress responses. J Psychosom Res. 2008;65(3):229-38.

29. Elie $M$, Cole MG, Primeau FJ, Bellavance F. Delirium risk factors in elderly hospitalized patients. J Gen Intern Med. 1998;13(3):204-12.

30. Shah FA, Girard TD, Yende S. Limiting sedation for patients with acute respiratory distress syndrome - time to wake up. Curr Opin Crit Care. 2017;23(1):45-51.
31. Hager DN, Dinglas VD, Subhas S, Rowden AM, Neufeld KJ, Bienvenu OJ, Touradji P, Colantuoni E, Reddy DR, Brower RG, et al. Reducing deep sedation and delirium in acute lung injury patients: a quality improvement project. Crit Care Med. 2013;41(6):1435-42.

32. Long L, Ren S, Gong Y, Zhao H, He C, Shen L, Zhao H, Ma P. Different depths of sedation versus risk of delirium in adult mechanically ventilated patients: A systematic review and meta-analysis. PLoS ONE. 2020;15(7):e0236014.

33. Leslie DL, Zhang Y, Holford TR, Bogardus ST, Leo-Summers LS, Inouye SK. Premature death associated with delirium at 1-year follow-up. Arch Internal Med. 2005;165(14):1657-62.

34. Kiely DK, Marcantonio ER, Inouye SK, Shaffer ML, Bergmann MA, Yang FM, Fearing MA, Jones RN. Persistent delirium predicts greater mortality. J Am Geriatr Soc. 2009;57(1):55-61.

35. Mulligan O, Muresan L, Murray O, Adamis D, McCarthy G. Mortality at one year post delirium in general medical inpatients. Eur Psychiatry. 2015;30(S1):1-1.

36. Wolters AE, van Dijk D, Pasma W, Cremer OL, Looije MF, de Lange DW, Veldhuijzen DS, Slooter AJ. Long-term outcome of delirium during intensive care unit stay in survivors of critical illness: a prospective cohort study. Crit Care. 2014;18(3):R125

37. Sanchez D, Brennan K, Al Sayfe M, Shunker SA, Bogdanoski T, Hedges S, Hou YC, Lynch J, Hunt L, Alexandrou E, et al. Frailty, delirium and hospital mortality of older adults admitted to intensive care: the Delirium (Deli) in ICU study. Crit Care. 2020;24(1):609.

\section{Publisher's Note}

Springer Nature remains neutral with regard to jurisdictional claims in published maps and institutional affiliations.
Ready to submit your research? Choose BMC and benefit from:

- fast, convenient online submission

- thorough peer review by experienced researchers in your field

- rapid publication on acceptance

- support for research data, including large and complex data types

- gold Open Access which fosters wider collaboration and increased citations

- maximum visibility for your research: over $100 \mathrm{M}$ website views per year

At BMC, research is always in progress.

Learn more biomedcentral.com/submissions 